\title{
How do you use bispectral index effectively for preventing re-awareness during general anesthesia?
}

\author{
Won Joon Choi, and Yun Hong Kim \\ Department of Anesthesiology and Pain Medicine, Sungkyunkwan University School of Medicine, Kangbuk Samsung Hospital, Seoul, \\ Korea
}

Low incidence $(0.1-0.4 \%)[1,2]$ awareness during intended general anesthesia brings significant postoperative sequelae to the patient, including sleep disturbances, nightmares, daytime anxiety, and even late psychological symptoms [3]. Traditionally, the detection of intraoperative awareness has been conducted by observing patient movement or developments of tachycardia and hypertension. However, these approaches are often hindered by anesthetics and supportive medicines used during general anesthesia and various surgical events. Recently, the Bispectral index (BIS), a mathematically derived electroencephalographic (EEG) derivative [4], has been adopted during general anesthesia to monitor anesthetic depth. By maintaining the BIS between 40 and 60, which is the manufacturer's recommended value for general anesthesia, a reduction of anesthetic requirement and shorter length of stay in PACU can be achieved [5]. However, a large-scale prospective study ( $\mathrm{n}=967$ ) reveals that the incidence rate for anesthesia awareness is $0.62 \%$ of BIS protocol based monitoring during general anesthesia [6]. Therefore, we need to consider which method is desirable to prevent re-awareness during general anesthesia using BIS monitoring. Seol et al. [7], in this issue of the Korean Journal of Anesthesiology, suggests the role of BIS for monitoring intraoperative awareness, although this is not the main theme of the article. In this present study, when the patients were forced back to awareness during general anesthesia with sevoflurane or desflurane, the mean BIS value at the first response to verbal command was about 90 . If the BIS value corresponding to the response to verbal command during anesthetic induction closely parallels that at the time of re-awareness during anesthesia, we can use the BIS values of anesthetic induction for monitoring anesthetic awareness. In the study using propofol, the BIS value was between 51 and 85 (median 67) at the point of transition to re-awareness from loss of response to verbal command [8]. In addition, in the course of induction by propofol, the range of BIS when maintaining the response to verbal command was similar to that of re-awareness from loss of consciousness: between 57 and 88 (median about 80) [9]. However, the median BIS value seemed to be higher during re-awareness than during induction. Further research is necessary to determine if the BIS value in the course of induction is an available parameter to detect re-awareness during surgery under general anesthesia.

Furthermore, to use BIS effectively, we should also consider the degree of muscle paralysis. It is known that the BIS value reflects not only electroencephalographic (EEG) activity but also electromyographic (EMG) activity of the facial muscles [10]. After administering muscle relaxants without any sedatives, the BIS value dropped to between 9 and 64, accompanied by the decline of EMG activity. Also, these variations of the BIS decline might be dependent on the degree of neuromuscular blockade. In the study by Seol et al. [7], a wake-up test was not conducted until confirming neuromuscular full recovery by a nerve stimulator. For this reason, BIS related to awareness might record a high value at about 90 . Paradoxically, the published reports of awareness have increased since 1990, even though there have been remarkable advancements in

Corresponding author: Yun Hong Kim, M.D, Ph.D., Department of Anesthesiology and Pain Medicine, Sungkyunkwan University School of Medicine, Kangbuk Samsung Hospital, Pyeong-dong, Jongno-gu, Seoul 110-746, Korea. Tel: 82-2-2001-2315, Fax: 82-2-2001-2326, E-mail: yhkim12@yahoo.co.kr

(c) This is an open-access article distributed under the terms of the Creative Commons Attribution Non-Commercial License (http:// creativecommons.org/licenses/by-nc/3.0/), which permits unrestricted non-commercial use, distribution, and reproduction in any medium, provided the original work is properly cited. 
the field of anesthesiology [3]. Based on the complaints of the patients with unwanted awareness, the universal use of muscle relaxants during surgical procedures may be closely associated with the increasing incidence of awareness. Also, episodes of awareness have been reported most frequently during anesthetic maintenance, when the neuromuscular block is usually sustained for a suitable surgical condition. Muscle paralytic degrees are changeable during maintenance in each patient because of the variable pharmacokinetic and pharmacodynamic characters of muscle relaxants. Therefore, the variable influences of the current EMG component on the BIS value should be considered when interpreting the values. If a neuromuscular block is conducted, the recommended BIS value would not fully guarantee complete hypnosis. According to the article by Avidan et al. [6], anesthesia awareness was reported at an incidence rate of $0.31 \%$ when the minimum alveolar concentration (MAC) value of the anesthetics used was maintained between $0.7-1.3$. This data was lower than that of the BIS monitoring based anesthesia, although there was no statistical significance. Therefore, in the cases of using muscle relaxants, BIS monitoring along with MAC based anesthetic management might be desirable.

In conclusion, considerable care is necessary in the interpretation of the BIS value when muscle relaxants are administered. Henceforth, it may be necessary to investigate the validity of using the BIS value during the induction period for detecting intraoperative re-awareness.

\section{References}

1. Sebel PS, Bowdle TA, Ghoneim MM, Rampil IJ, Padilla RE, Gan TJ, et al. The incidence of awareness during anesthesia: a multicenter United States study. Anesth Analg 2004; 99: 833-9.

2. Xu L, Wu AS, Yue Y. The incidence of intra-operative awareness during general anesthesia in China: a multi-center observational study. Acta Anaesthesiol Scand 2009; 53: 873-82.

3. Ghoneim MM, Block RI, Haffarnan M, Mathews MJ. Awareness during anesthesia: risk factors, causes and sequelae: a review of reported cases in the literature. Anesth Analg 2009; 108: 527-35.

4. Rampil IJ. A primer for EEG signal processing in anesthesia. Anesthesiology 1998; 89: 980-1002.

5. Recart A, Gasanova I, White PF, Thomas T, Ogunnaike B, Hamza $\mathrm{M}$, et al. The effect of cerebral monitoring on recovery after general anesthesia: a comparison of the auditory evoked potential and bispectral index devices with standard clinical practice. Anesth Analg 2003; 97: 1667-74.

6. Avidan MS, Zhang L, Burnside BA, Finkel KJ, Searleman AC, Selvidge JA, et al. Anesthesia awareness and the bispectral index. N Engl J Med 2008; 358: 1097-108.

7. Seol TK, Han MK, Lee HJ, Cheong MA, Jun JH. Bispectral index and their relation with consciousness of the patients who receive desflurane or sevoflurane anesthesia during wake-up test for spinal surgery for correction. Korean J Anesthesiol 2012; 62: 13-8.

8. Barr G, Anderson RE, Owall A, Jakobsson JG. Being awake intermittently during propofol-induced hypnosis: a study of BIS, explicit and implicit memory. Acta Anaesthesiol Scand 2001; 45: 834-8.

9. Nishiyama T. Auditory evoked potentials index versus bispectral index during propofol sedation in spinal anesthesia. J Anesth 2009; 23: 26-30.

10. Messner M, Beese U, Romstöck J, Dinkel M, Tschaikowsky K. The bispectral index declines during neuromuscular block in fully awake persons. Anesth Analg 2003; 97: 488-91. 